\title{
Management of Chronic Subdural Hematoma in Patients with Intractable Thrombocytopenia
}

\author{
Mohamed ABDELFATAH \\ Ain Shams University, Department of Neurosurgery, Cairo, Egypt
}

\section{ABSTRACT}

AIM: Currently, the platelet count threshold for platelet transfusion, that is commonly used prior to surgical evacuation of chronic subdural hematoma (CSDH), is 100,000 per microliter. This study was done to show the outcome of the surgical evacuation of CSDH in patients with intractable thrombocytopenia (platelet count which did not reach 100,000 per microliter after platelet transfusions).

MATERIAL and METHODS: This study retrospectively reviewed the data of patients who were admitted to our university hospitals with symptomatic CSDH and intractable thrombocytopenia from January 2007 to January 2016.

RESULTS: Forty-one patients were included in this study. There was no incidence of intraoperative or postoperative acute bleeding in any patient.

CONCLUSION: Evacuation of CSDH in thrombocytopenic patients, without reaching a platelet count of 100,000 per microliter, is an option. Further randomized controlled trials are needed to determine the correct platelet count threshold prior to evacuation of $\mathrm{CSDH}$.

KEYWORDS: Chronic subdural hematoma, Intractable thrombocytopenia, Burr hole, Platelet transfusion

\section{INTRODUCTION}

$(2$ hronic subdural hematoma $(\mathrm{CSDH})$ is a common situation in neurosurgical practice. The treatment of choice for a symptomatic chronic subdural hematoma is its urgent surgical evacuation (8).

Evacuation of a chronic subdural hematoma is not a major neurosurgical operation as no major blood loss is expected and no prolonged general anesthesia is required. It can also be performed under local anesthesia.

The standard of practice nowadays is to administer prophylactic platelet transfusions before major neurosurgical operations if the platelet count is below the threshold of 100,000 per microliter to decrease the incidence of acute bleeding (6). However, in emergency operations, the platelet count should be at least 80,000 per microliter.
This study was done to show the outcome of the surgical evacuation of CSDH in patients with intractable thrombocytopenia (platelet count which did not reach 100,000 per microliter after platelet transfusions).

\section{MATERIAL and METHODS}

This study retrospectively reviewed the data of patients who admitted to our university hospital with symptomatic CSDH and intractable thrombocytopenia from January 2007 to January 2016. The aim of the study was to show their surgical outcome.

Inclusion criteria:

- Patients with symptomatic CSDH

- Patients with platelet count on admission <100,000/ microliter.

- Patients of any age or gender. 
Exclusion criteria:

- Patients with CSDH who did not undergo surgical evacuation.

- Patients with ethylenediaminetetraacetic acid (EDTA)induced thrombocytopenia.

- Thrombocytopenic patients whose preoperative platelet counts reached 100,000 per microliter by platelet transfusions.

- Patients with decompensated liver cirrhosis and acute liver failure.

The clinical data were collected from the patients' charts and include; history of head trauma, associated medical diseases, the etiology of thrombocytopenia, the preoperative clinical state, laboratory findings, preoperative computed tomography (CT) scan findings, the preoperative preparation, time between admission and surgery, full operative details, postoperative management, any complications from surgery, follow-up CT scan findings and the progressive notes in the follow-up visits.

The neurological condition of the patients was preoperatively and postoperatively evaluated with the Markwalder's Neurological Grading System (Table I) (7).

The type of CSDH was classified on CT scan as mixed density containing some acute blood, isodensity to the brain, or hypodensity to the brain. Clot volume was measured by multiplying the maximum extent in each of 3 orthogonal views and dividing by 2 .

On admission, all patients started a prophylactic antiepileptic in the form of phenytoin (loading and maintenance doses) except in known hepatic patients who were started levetiracetam instead of phenytoin.

Patients received a prophylactic intravenous antibiotic (ceftriaxone) within one hour of the operation.

In the operating theater, patients received general anesthesia with a muscle relaxant. Prep was done using 7.5\% Povidone lodine for cleaning and $10 \%$ Povidone lodine for painting.

Two linear scalp incisions down to bone were done on the maximal thickness of the subdural hematoma. One burr hole in each scalp incision was done with a diameter of at least $1.5 \mathrm{~cm}$. Incision of the dura and washing the subdural space by warm saline was done till the wash becomes clear. One subgaleal drain for each scalp wound was inserted and then was removed two to three days post-op.

No subdural drain was used in any case.

The operated patients were flat in bed for 2 days after the operation then had a gradual elevation of the head.

All patients had received a preoperative platelet transfusion of 18 units of random donor pooled (RDP) platelets but did not reach the platelet count of 100,000 per microliter.

All patients received intraoperatively 6 units of RDP platelet transfusion and another 6 units postoperatively within 12 hours of surgery.
Follow-up cranial CT scan was done for all patients after 48 hours of the operation, after one month and after 6 months of surgery.

Follow-up period was at least 12 months after surgery (ranging from 12 months to 26 months)

\section{- RESULTS}

Forty-one patients with symptomatic CSDH and intractable thrombocytopenia were included in this study.

The age of the patients in this study ranged from 28 to 84 years with a mean age of 59 years. Twenty-four patients (58.5\%) were male.

Twenty-six patients (63.4\%) presented in Markwalder's grade 1. Nine patients $(21.9 \%)$ presented in Markwalder's grade 2, and six patients (14.6\%) presented in Markwalder's grade 3 and none in grade 4.

Thirty-four patients (82.9\%) had a history of head trauma (apparently trivial trauma) within 6 weeks before admission, without any following alteration in consciousness.

Associated medical diseases and the etiology of thrombocytopenia are seen in Tables II and III.

All patients had a prior history of platelet transfusion. All patients were not on any anti-platelets or anti-coagulants.

Thirty-three patients had isolated thrombocytopenia and eight patients had pancytopenia.

The platelet count on admission of the patients in this study ranged from 23,000 to 62,000 per microliter (Table IV).

The type of CSDH was classified on CT scan as mixed density containing some acute blood, isodense to the brain, or hypodense to the brain. Twenty-three patients had mixed density $\mathrm{CSDH}$, eleven patients had isodense $\mathrm{CSDH}$, and seven patients had hypodense $\mathrm{CSDH}$.

Clot volume ranged between 55 to $132 \mathrm{~cm}^{3}$ with a mean volume of $81 \mathrm{~cm}^{3}$.

The mean thickness of the CSDH on the CT scan was $2.4 \mathrm{~cm}$, ranging from 1.6 to $3.4 \mathrm{~cm}$. The midline shift ranged between $0.6 \mathrm{~mm}$ to $2.3 \mathrm{~cm}$ with a mean shift of $1.4 \mathrm{~cm}$.

CSDH was bilateral in 4 cases (9.7\%).

Eight patients received packed $\mathrm{RBCs}$ transfusion to raise the hemoglobin to at least $10 \mathrm{gm} / \mathrm{dl}$.

Nine patients received fresh frozen plasma (FFP) transfusions to decrease the international normalized ratio (INR) to at least 1.4 .

All patients included in this study received preoperatively 6 units of RDP platelets to raise the platelet count and a new platelet count was done 30 minutes after the transfusion. This was repeated three times without correction of the platelet count to the cut-off value of 100,000 per microliter (Table IV).

The surgical evacuation was decided with intraoperative and postoperative platelet transfusions. The immediate 
Table I: Markwalder's Neurological Grading System (7)

\begin{tabular}{cl}
\hline Grade & State of the patient \\
\hline $\mathbf{0}$ & Patient neurologically normal \\
\hline $\mathbf{1}$ & $\begin{array}{l}\text { Patient alert and oriented; mild symptoms, such as headache; absent or mild symptoms or neurological deficit, such } \\
\text { as reflex asymmetry }\end{array}$ \\
\hline $\mathbf{2}$ & Patient drowsy or disoriented with variable neurological deficit, such as hemiparesis \\
\hline $\mathbf{3}$ & Patient stuporous, but responding appropriately to noxious stimuli; several focal signs, such as hemiparesis \\
\hline $\mathbf{4}$ & Patient comatose with absent motor response to painful stimuli; decerebrate or decorticate posturing. \\
\hline
\end{tabular}

Table II: Medical Diseases

\begin{tabular}{lc}
\hline Medical disease & Number of patients \\
\hline DM & 21 \\
\hline Hypertension & 24 \\
\hline Hepatitis C virus & 24 \\
\hline Cirrhosis & 9 \\
\hline Chronic ITP & 8 \\
\hline Old non metastatic Breast cancer & 2 \\
\hline
\end{tabular}

ITP: idiopathic thrombocytopenic purpura, DM: diabetes mellitus.

Table III: Etiology of Thrombocytopenia

\begin{tabular}{|c|c|}
\hline Etiology of thrombocytopenia & Number of patients \\
\hline $\begin{array}{l}\text { Direct myelosuppressive effects of } \\
\text { HCV infection }\end{array}$ & 24 \\
\hline Cirrhosis & 9 \\
\hline Chronic ITP & 8 \\
\hline
\end{tabular}

HCV: Hepatitis C virus.

preoperative platelet count for the patients is listed in Table IV. The duration between admission and surgery ranged from 47 to 85 hours.

The duration of surgery (from skin incisions to skin closure) ranged between 51 minutes to 86 minutes with a mean duration of 55 minutes. All surgeries were uneventful.

The follow-up cranial CT scan, which was done 48 hours after the operation, showed a decrease in the size of the CSDH, decrease in midline shift with a variable amount of air in the subdural space and no acute subdural hematoma.

All operated patients improved clinically within few days after surgery.

Routine follow-up CT scan at one month after surgery showed no recurrence of $\mathrm{CSDH}$, except in two patients who had recurrence of $\mathrm{CSDH}$ and had re-evacuation via another burr holes.
Routine follow-up CT scan after 6 months of surgery showed no recurrence of $\mathrm{CSDH}$.

No postoperative symptomatic acute subdural hematoma or intracerebral hematoma occurred in any patient in this study.

No patient developed an infection within the surgical field during the whole period of follow-up.

Follow-up period was at least 12 months after surgery (ranging from 12 months to 26 months).

\section{DISCUSSION}

Platelets play a vital role in hemostasis. Platelet transfusion plays a significant value for thrombocytopenic patients in preventing hemorrhage. There are no randomized controlled trials on the exact platelet count which permits a safe cranial surgery, but there is an expert opinion to keep the preoperative platelet count at least 100,000/microliters.

The American Association of Blood Banks (AABB) in 2015 recommends platelet transfusion of up to a single unit of apheresis platelets or equivalent and added that extra doses are not more effective (3). This dose may increase the platelet count by $60,000 /$ microliter within a peak of one hour (1).

In this study, 41 thrombocytopenic patients had a surgical evacuation of their symptomatic CSDH. These patients received preoperative platelet transfusion but their thrombocytopenia was intractable i.e. their platelet count didn't reach 100,000 per microliter.

Although many thrombocytopenic patients respond appropriately after platelet transfusion, inadequate correction tends to occur and fail to produce a satisfactory response. This intractable thrombocytopenia leads to longer hospital stays and higher inpatient hospital costs $(4,5)$.

All patients in this study improved clinically within few days after surgery, and the follow-up cranial CT scan, done 48 hours after the operation, showed a decrease in the size of the $\mathrm{CSDH}$, and decrease in midline shift in all patients.

Two patients had a recurrence of CSDH after one month of surgery and had re-evacuation. The cause of recurrence was probably because of the plenty of septae in the CSDH.

No postoperative acute subdural hematoma or intracerebral hematoma occurred in any patient in this study. 
Table IV: Platelet Counts

\begin{tabular}{|c|c|c|}
\hline Patient No & Platelet count on admission (per microliter) & Last preoperative Platelet count (per microliter) \\
\hline 1 & 27000 & 51000 \\
\hline 2 & 28000 & 54000 \\
\hline 3 & 31000 & 59000 \\
\hline 4 & 36000 & 56000 \\
\hline 5 & 23000 & 54000 \\
\hline 6 & 28000 & 69000 \\
\hline 7 & 29000 & 58000 \\
\hline 8 & 60000 & 72000 \\
\hline 9 & 39000 & 56000 \\
\hline 10 & 32000 & 66000 \\
\hline 11 & 43000 & 77000 \\
\hline 12 & 51000 & 65000 \\
\hline 13 & 55000 & 71000 \\
\hline 14 & 62000 & 79000 \\
\hline 15 & 34000 & 63000 \\
\hline 16 & 23000 & 55000 \\
\hline 17 & 54000 & 77000 \\
\hline 18 & 26000 & 57000 \\
\hline 19 & 61000 & 74000 \\
\hline 20 & 36000 & 70000 \\
\hline 21 & 49000 & 66000 \\
\hline 22 & 56000 & 69000 \\
\hline 23 & 34000 & 55000 \\
\hline 24 & 35000 & 62000 \\
\hline 25 & 46000 & 75000 \\
\hline 26 & 39000 & 51000 \\
\hline 27 & 50000 & 71000 \\
\hline 28 & 38000 & 64000 \\
\hline 29 & 49000 & 61000 \\
\hline 30 & 29000 & 66000 \\
\hline 31 & 51000 & 73000 \\
\hline 32 & 31000 & 66000 \\
\hline 33 & 60000 & 73000 \\
\hline 34 & 54000 & 76000 \\
\hline 35 & 41000 & 65000 \\
\hline 36 & 45000 & 67000 \\
\hline 37 & 49000 & 61000 \\
\hline 38 & 32000 & 54000 \\
\hline 39 & 59000 & 68000 \\
\hline 40 & 32000 & 61000 \\
\hline 41 & 43000 & 71000 \\
\hline
\end{tabular}


Conducting a major neurosurgical operation in a patient with a platelet count $<100,000 /$ microliters carries a high degree of risk. Chan et al. observed that there is a high risk of postoperative hematoma in thrombocytopenic patients with preoperative platelet count below 100,000/microliters (2).

The limitations of this study are many; a retrospective study, a small number of patients, and absence of control group, but this study opens our mind that further randomized controlled trials are necessary to determine the correct platelet count threshold prior to safe evacuation of $\mathrm{CSDH}$.

\section{- CONCLUSION}

Evacuation of CSDH in thrombocytopenic patients, without reaching a platelet count of 100,000 per microliter, is an option.

\section{- REFERENCES}

1. Blumberg N, Heal JM, Phillips GL: Platelet transfusions: Trigger, dose, benefits, and risks. F1000 Med Rep 2: 5, 2010

2. Chan KH, Mann KS, Chan TK: The significance of thrombocytopenia in the development of postoperative intracranial hematoma. J Neurosurg 71 (1):38-41, 1989
3. Kaufman RM, Djulbegovic B, Gernsheimer T, Kleinman S, Tinmouth AT, Capocelli KE, Cipolle MD, Cohn CS, Fung MK, Grossman BJ, Mintz PD, O'Malley BA, Sesok-Pizzini DA, Shander A, Stack GE, Webert KE, Weinstein R, Welch BG, Whitman GJ, Wong EC, Tobian AA; AABB: Platelet transfusion: A clinical practice guideline from the AABB. Ann Intern Med 162:205-213, 2015

4. Kerkhoffs JL, Eikenboom JC, van de Watering LM, van Wordragen-Vlaswinkel RJ, Wijermans PW, Brand A: The clinical impact of platelet refractoriness: Correlation with bleeding and survival. Transfusion 48:1959-1965, 2008

5. Legler TJ, Fischer I, Dittmann J, Simson G, Lynen R, Humpe A, Riggert J, Schleyer E, Kern W, Hiddemann W, Köhler M: Frequency and causes of refractoriness in multiply transfused patients. Ann Hematol 74:185-189, 1997

6. Liumbruno G, Bennardello F, Lattanzio A, Piccoli P, Rossetti G: Recommendations for the transfusion of plasma and platelets. Blood Transfus 7:132-150, 2009

7. Markwalder TM, Steinsiepe KF, Rohner M, Reichenbach W, Markwalder $\mathrm{H}$ : The course of chronic subdural hematomas after burr-hole craniostomy and closed-system drainage. J Neurosurg 55: 390-396, 1981

8. Okada Y, Akai T, Okamoto K, lida T, Takata H, lizuka H: A comparative study of the treatment of chronic subdural hematoma; Burr hole drainage versus burr hole irrigation. Surg Neurol 57:405-409, 2002 